PAPER

\title{
Reliability of self-reported diagnoses in patients with neurologically unexplained symptoms
}

\author{
A Schrag, R J Brown, M R Trimble
}

J Neurol Neurosurg Psychiatry 2004;75:608-611. doi: 10.1136/jnnp.2003.020552

See end of article for authors' affiliations

....... a r.

Correspondence to: Dr A Schrag, Division of Neuropsychiatry and

Neuropsychology, Institute of Neurology, Queen Square, London WCIN 3BG; a.schrag@

ion.ucl.ac.uk

Received 11 June 2003

In revised form

6 August 2003

Accepted 16 October 2003
Background: Patients with neurologically unexplained symptoms (NUS) often have a previous history of other medically unexplained symptoms. A past history of such symptoms can help make a positive diagnosis of a somatoform or affective disorder, and enable appropriate management strategies. However, information on past medical diagnoses is primarily obtained from patient interviews and may be inaccurate, particularly in patients with NUS.

Objective: To assess the reliability of past medical diagnoses reported by patients with NUS compared with patients with confirmed neurological disease (ND) without suspicion of somatoform illness.

Methods: 21 patients with NUS and 16 patients with ND were interviewed about their current and past medical problems and diagnoses. The accuracy of the reported diagnoses was assessed through examination of their complete general practice notes.

Results: The median number of previous diagnoses reported by patients with NUS was significantly higher than in controls $(7 \vee 3, p=0.001)$. There was no difference in the median number of confirmed diagnoses between the two groups ( $2 \vee 2.5)$; however, the median percentage of reported diagnoses confirmed by investigations was significantly smaller in the NUS group $(22 \% \vee 80 \%, p=0.001)$. The additional diagnoses reported by patients with NUS not only comprised functional syndromes such as irritable bowel syndrome or non-cardiac chest pain $(6 \% \vee 0 \%, p=0.01)$, but also organic diagnoses which had either been unequivocally excluded $(5 \% \vee 0 \%, p=0.006)$, were based on equivocal findings often found after multiple investigations $(9 \% \vee 0 \%, p=0.01)$, or had not been investigated before a clinical diagnosis was made $(50 \%$ v 18\%, $p=0.04)$.

Conclusion: Reported previous diagnoses should not be taken at face value when the current differential diagnosis includes a functional/somatoform neurological syndrome, particularly if the list of past medical diagnoses is long. Confirmation of previous diagnoses from alternative sources may contribute to a diagnosis of somatoform disorder, allowing appropriate management strategies for the current (and past) complaints to be initiated.

\section{INTRODUCTION}

Ascertaining an accurate past medical history ( $\mathrm{PMH})$ is a routine part of clinical assessment, and may have implications for the diagnosis of a new complaint. Unless there is significant cognitive impairment, the primary informant of the $\mathrm{PMH}$ is the patient. This information is often complemented by the referring doctor's report of the PMH, but this also relies on information provided by the patient. Patients with neurologically unexplained symptoms (NUS) often have had other medically unexplained symptoms, ${ }^{12}$ but may not have acquired a diagnosis of somatoform disorder, which is frequently underdiagnosed. ${ }^{3}$ Knowledge of a prior history of medically unexplained symptoms can help make a positive diagnosis of a somatoform or affective disorder, prevent overdiagnosis and medicalisation of somatic symptoms, avoid unnecessary investigations and treatments, and enable appropriate intervention. In this study, we assessed the reliability of patient-reported past medical diagnoses in patients with NUS in the context of unequivocal somatoform illness compared with a control group of patients with a confirmed neurological diagnosis. We hypothesised that patients with NUS would not only report larger numbers of past medical diagnoses, but that many of these would not have been confirmed by investigations.

\section{PATIENTS AND METHODS}

Patients with NUS who fulfilled a clinical diagnosis of multisomatoform disorder, defined as three or more medically unexplained, currently bothersome physical symptoms plus a long ( $\geqslant 2$ years) history of somatisation, ${ }^{4}$ were recruited from the National Hospital for Neurology and Neurosurgery (NHNN). All patients with NUS had received inpatient or outpatient treatment at the NHNN and had been intensively investigated for their presenting neurological symptoms, for which no organic cause had been found. A total of 27 patients with NUS agreed to participate, of whom 11 were identified retrospectively through analysis of discharge summaries from the hospital neuropsychiatry ward for the preceding three years, and 16 were identified prospectively following admission to a neurological or neuropsychiatry ward and had been identified as suffering from multiple unexplained symptoms during this admission. From these, complete records were available for 21 patients whose presenting neurological symptoms included involuntary movements $(n=7)$, nonepileptic seizures $(n=2)$, sensorimotor hemiparesis $(n=2)$, leg weakness $(n=3)$, back pain with weakness of one or both legs associated with blurred vision and slurred speech $(n=2)$, fatigue and speech disturbance $(n=1)$, attacks of unsteadiness and slurred speech $(\mathrm{n}=3)$, and whole body numbness $(\mathrm{n}=1)$. The diagnosis of somatoform disorder had been made without use of the primary care records. Twenty

Abbreviations: DVT, deep vein thrombosis; GP, general practice; IBS, irritable bowel syndrome; ND, neurological disorder; NUS, neurologically unexplained symptoms; PMH, past medical history 
one patients without suspicion of medically unexplained symptoms were recruited as controls (neurological disorder (ND) group) from a neurological botulinum toxin clinic. Complete records were available for 16. They had a diagnosis of primary dystonia $(\mathrm{n}=2$ cervical dystonia; $\mathrm{n}=3$ generalised dystonia due to the DYT 1 mutation) or secondary dystonia ( $\mathrm{n}=5$ due to a basal ganglia lesion; $\mathrm{n}=6$ due to anoxic birth injury), and were matched for age, sex and physician-rated overall impression of disability with the NUS group. Patients with significant cognitive impairment were excluded. All patients gave informed, written consent, and the study, which was part of a larger study on NUS, was approved by the Joint Medical Ethics committee of the Institute of Neurology and the National Hospital for Neurology and Neurosurgery.

All patients underwent an open interview about their current and past medical history, and a structured interview about previous physical symptoms and disorders as part of the Schedules of Assessment in Neuropsychiatry (SCAN). ${ }^{5}$ We examined the general practice (GP) notes of all patients who consented and for whom complete GP records were available. In the UK, GPs hold all medical records of patients, and examination of these records, where complete and available, permits a comprehensive summary of each patient's current and past medical problems, investigation results, and treatments.

The diagnoses reported by patients were compared with the information obtained from GP records and hospital notes. Patient-reported diagnoses were classified into five groups:

- appropriately investigated with conclusive, confirmatory results of an organic diagnosis-for example, colonoscopy confirmed diverticular disease

- appropriately investigated and no cause found-for example, angina pectoris with normal angiogram

- appropriately investigated and investigations inconclusive or showing equivocal findings-for example, epilepsy with non-specific electroencephalogram (EEG) changes

- diagnosis made on clinical grounds-for example, a diagnosis of deep vein thrombosis (DVT), or sciatica; or a symptom leading to treatment without firm diagnosisfor example, hysterectomy for menorrhagia without obvious cause

- diagnosis of a "functional somatic syndrome" including irritable bowel syndrome (IBS), non-ulcer dyspepsia, fibromyalgia, non-cardiac chest pain, and chronic fatigue syndrome.

GP consultations for repeat prescriptions, advice, or minor medical symptoms such as pharyngitis, otitis media, or a single episode of gastroenteritis were not included. The complaints for which the patients were referred to the NHNN, and the diagnoses given for these, were excluded from the analysis.

The rates of reported past medical diagnoses in each category of diagnostic certainty in the two groups of patients were compared. As most data were not normally distributed, medians and ranges were calculated. Categorical variables were compared using $\chi^{2}$ tests, and continuous variables using the Mann-Whitney U test.

\section{RESULTS}

One patient in the ND group refused access to their records, the GP refused access or requested a fee beyond the budget of the study $(>£ 70)$ for the records of four patients in each group, and the records were untraceable in two patients with NUS. We were therefore able to examine the GP records of 21 of the patients with NUS and 16 patients with ND; complete hospital notes were also available for all of these patients. The patients whose GP records we were able to obtain did not differ in age, sex or diagnostic group from those for whom this was not possible. There was no difference in the mean (SD) age of patients with NUS and ND (42.0 (10.6) years $v 45.4$ (14.9) years). Five NUS patients in the NUS and four in the ND group were male. This difference was nonsignificant. The GP records reached back over an average (SD) time of 31.9 (10.5) years to a mean start age of 11.3 (10.5) years.

Patients with NUS reported significantly more previous medical diagnoses than controls $(\mathrm{n}=7 v \mathrm{n}=3, \mathrm{p}=0.001$; see table 1). The total number of confirmed medical diagnoses was not significantly different between the two groups $(\mathrm{n}=2$ $v \mathrm{n}=2.5$ ); however, the percentage of reported diagnoses confirmed by investigations was significantly smaller in the NUS group $\left(22 \% v 80 \%, \mathrm{p}=0.001^{*}\right)$. NUS patients reported significantly more diagnoses that had either been (i) definitely excluded by appropriate investigations (5\% $\vee 0 \%$, $\mathrm{p}=0.006$ ); (ii) were unconfirmed by often extensive investigations, which had shown equivocal results (9\% $\vee 0 \%$, $\mathrm{p}=0.01$ ); or (iii) were based on clinical judgement in the absence of laboratory investigations $(50 \% v 18 \%, \mathrm{p}=0.04)$. In addition, they reported significantly higher numbers of functional somatic syndromes, such as IBS or non-cardiac chest pain $(6 \% v 0 \%, \mathrm{p}=0.01)$, diagnoses made following the exclusion of organic causes.

Reported diagnoses that had been unequivocally excluded conformed to no specific medical specialty and included appendicitis, meningitis, multiple sclerosis, epilepsy, and angina pectoris. Diagnoses reported but only supported by equivocal abnormalities on investigations included colitis and Addison's disease. Diagnoses that were based on clinical features only, but were treated, included pain syndromes for which no cause was found, DVT, eczema, asthma and menorrhagia. The diagnoses most difficult to categorise were those made on clinical grounds alone. Many of the diagnoses reported in our sample-for example, DVTs could have been confirmed or excluded had they been investigated; others had unusual features suggestive of a non-organic aetiology, such as menorrhagia leading to hysterectomy at a young age, asthma with very fluctuating peak flow rates, and "epileptic attacks" always triggered by stress and typically associated with tetany.

In our sample, both clinical diagnoses without confirmatory investigations, and abnormal findings of equivocal significance to the presenting complaint, often led to invasive or long term treatments. For example, the finding of an ovarian cyst in a patient investigated for constant abdominal pain led to the removal of the cyst, under general anaesthetic, without subsequent improvement of symptoms. Another patient had been on long term anticonvulsant treatment for non-confirmed epileptic seizures. Two patients had been given short courses of steroids, one for an equivocal diagnosis of adrenal insufficiency, and the second for an excluded diagnosis of multiple sclerosis. A number of patients were on folic acid supplements or on specific diets for questionable diagnoses of diabetes mellitus or multiple food allergies. Some patients had undergone potentially unnecessary operations such as hysterectomies or appendicectomies with no histological abnormality found at operation. Finally, some patients had developed symptoms due to confirmed abnormalities, resulting from previous possibly inappropriate investigation or treatment; thus one patient had developed chronic abdominal pain related to adhesions following ovarian

*Note that the apparent error in percentages is the result of calculating median percentages, which differ slightly from the percentages of the overall medians. 
Table 1 Diagnostic certainty of reported past medical diagnoses

\begin{tabular}{|c|c|c|c|}
\hline & $\begin{array}{l}\text { Patients with NUS }(n=21) \\
\text { Median (range) }\end{array}$ & $\begin{array}{l}\text { Patients with ND ( } n=16) \\
\text { Median (range) }\end{array}$ & $\mathrm{p}$ value \\
\hline Patient-reported diagnoses & $7(2-23)$ & $3(1-14)$ & 0.001 \\
\hline Confirmed by investigations & $2(0-6)$ & $2.5(0-9)$ & \\
\hline Ruled out by investigations & $1(0-2)$ & $0(0)$ & 0.006 \\
\hline Investigations inconclusive & $1(0-3)$ & $0(0-1)$ & 0.01 \\
\hline Clinical diagnosis only & $3(0-15)$ & $1(0-5)$ & 0.001 \\
\hline Functional syndrome & $1(0-5)$ & $0(0-1)$ & 0.006 \\
\hline
\end{tabular}

cystectomy and an appendicectomy to alleviate acute abdominal pain.

\section{DISCUSSION}

We found support for our hypothesis that patients with NUS report larger numbers of previous medical diagnosis than patients with unequivocal neurological diagnoses. Although it is not surprising that patients with NUS fulfilling criteria for multisomatoform disorder had larger numbers of previous medically unexplained symptoms (as this is a requirement for the diagnosis ${ }^{4}$ ), or that they had more diagnoses of functional somatic syndromes than those with neurological disease, ${ }^{1}$ more than half of the apparently non-functional diagnoses reported by the NUS patients had not been confirmed by investigations; moreover, some had actually been firmly excluded. In addition, some diagnoses were based on equivocal investigation results but were reported as unequivocal diagnoses by the patients.

There are a number of possible explanations for these findings. It is possible that negative investigation results were explained to patients but were misunderstood or remembered incorrectly. This may be due to poor communication between doctor and patient, abnormal illness beliefs, lack of an alternative explanation, or the need to have an explanation for a troublesome symptom. Factors similar to those thought to be underlying the development of somatoform symptoms, such as illness experiences, ${ }^{6}$ somatic perception ${ }^{7}$ (including somatic preoccupation as part of a depressive syndrome ${ }^{8}$ ), attention, ${ }^{9}$ and cognitive evaluation, ${ }^{8}$ are also likely to play a role in the recollection and evaluation of past medical symptoms. It is possible that doctors may be more likely to reach a positive diagnosis based on borderline investigation results, or following no investigation at all, in this group. This may be due to the frequency with which this group of patients presents to their doctors. When confronted with a frequent attender, there may be a tendency either to make diagnoses on clinical grounds alone, or conversely to investigate each presenting symptom. The former may lead to inappropriate or excessive treatment, whilst the latter may lead to an increased likelihood of finding a borderline value or an abnormality not of relevance to the presenting symptom(s). Although assuming an organic cause is often considered a safer course of action, overdiagnosis is not without its problems. Particularly in neurology, overdiagnosis may carry the same risk as underdiagnosis, as many organic conditions have no specific treatments and the danger of iatrogenesis is considerable. It is notable that many patients with NUS in this series were still receiving treatment for unconfirmed diagnoses or had undergone invasive investigations during the diagnostic period. Furthermore, once a diagnosis is made (or even entertained) in this patient group, it may be very difficult to overturn.

Few comparable data on the reliability of reported $\mathrm{PMH}$ in patients with medically unexplained symptoms are available. In a recent study, patients with confirmed pseudoseizures had a significantly higher rate of self-reported asthma than patients with psychosis ${ }^{10}$; one of the possible interpretations offered by the authors was a misattribution of non-asthmatic respiratory symptoms to asthma. Wolfe and Hawley ${ }^{11}$ found that patients with fibromyalgia reported more comorbid conditions and attributed greater importance to these than patients with rheumatoid arthritis or osteoarthritis. It has also been shown that patients with NUS report significantly greater numbers of operations (particularly gynaecological procedures and appendicectomies) than healthy or medically ill controls. ${ }^{12}$ This may again be a direct consequence of the frequency with which these patients present with symptoms. Thus whilst uterine fibroids are present in more than $70 \%$ of women between 35 and 49 years, ${ }^{13}$ only a minority of these women will present to their doctors; of these, few will be investigated and have a diagnosis made, and fewer still will receive operative treatment. There may also be further problems in relying on self-reported symptoms in this patient group. In a longitudinal assessment of somatoform symptoms, $61 \%$ of lifetime medically unexplained symptoms were not reported after 12 months, and less than a third of patients fulfilling DSM IV criteria for somatisation disorder at baseline could be identified at follow up. ${ }^{14}$ These results and our findings underline the importance of obtaining accurate medical records relating to symptoms and diagnoses in these patients.

Our study has some limitations. As the current diagnosis was always listed in the GP notes, it was not possible to undertake a blinded retrospective review of these records. An observer bias cannot therefore be excluded as the investigator rating the GP records had knowledge of the patient's diagnosis. We were not able to assess the interobserver reliability of our classification of diagnosis as only one assessor examined the case notes; it is therefore possible that another assessor would have classified some diagnoses differently. However, we attempted to reduce this potential bias by categorising diagnoses into different degrees of diagnostic certainty, an approach supported by our finding of similar numbers of confirmed medical diagnoses in both groups. Nevertheless, it is possible that the knowledge that a patient has NUS may have led to an overestimation of previous symptoms as being medically unexplained. It should also be noted that our patient sample was small and represents a highly selected group of patients at the extreme end of a spectrum of somatoform illness. As patients were recruited from a tertiary referral centre with many patients fulfilling DSM IV criteria for somatisation disorder (requiring at least four unexplained pain, two gastrointestinal, one sexual and one pseudoneurological symptom with onset before age 30 years), it could be argued that our conclusions are not transferable to isolated non-organic symptoms. However, patients with functional somatic syndromes often have a history of other medically unexplained symptoms ${ }^{12}$ and this may only become apparent when the GP records are examined.

The results of this study suggest that neurologists presented with a patient with new neurological symptoms 
(particularly if the presentation is atypical) should keep an open mind as to the reliability of the past medical diagnoses reported by the patient, particularly if the list of previous diagnoses is unusually long. Since between $10 \%$ and $30 \%$ of patients presenting to neurological clinics will have NUS, ${ }^{15} 16$ this is a common problem in routine practice. Caution should be exercised in the interpretation of clinical diagnoses or those based on only equivocal investigation findings; moreover, the fact that a patient is receiving ongoing treatment for a condition does not reliably indicate that this disease has been definitively diagnosed. Once a diagnosis has been given (or even suspected) in patients with somatoform disorder, this may lead to concretisation of the disorder in the patient's mind, abnormal illness beliefs, perpetuation of illness behaviour and inappropriate, potentially harmful treatment. Knowledge of a history of previous medically unexplained symptoms may help make a positive diagnosis of a somatoform or affective disorder, prevent overdiagnosis and medicalisation of somatic symptoms, avert unnecessary investigations and treatments, and enable the initiation of appropriate management strategies.

\section{ACKNOWLEDGEMENTS}

We are grateful to the patients for participating in the study and to their general practitioners for providing access to their records. We thank Dr Jonathan Schott for his comments on an earlier draft of this paper.

\section{Authors' affiliations}

A Schrag, R J Brown, M R Trimble, Division of Neuropsychiatry and Neuropsychology, Institute of Neurology, London, UK

Competing interests: none declared

\section{REFERENCES}

1 Wessely S, Nimnuan C, Sharpe M. Functional somatic syndromes: one or many? Lancet 1999;354:936-9.

2 Stone J, Sharpe M, Rothwell PM, et al. The 12 year prognosis of unilateral functional weakness and sensory disturbance. J Neurol Neurosurg Psychiatry 2003;74:591-6.

3 Zimmerman M, Mattia Jl. Psychiatric diagnosis in clinical practice: is comorbidity being missed? Compr Psychiatry 1999;40:182-91.

4 Kroenke K, Spitzer RL, deGruy FV 3rd, et al. ultisomatoform disorder. An alternative to undifferentiated somatoform disorder for the somatizing patient in primary care. Arch Gen Psychiatry 1997;54:352-8.

5 Aboraya A, Tien A, Stevenson J, et al. Schedules for Clinical Assessment in Neuropsychiatry (SCAN): introduction to $\mathrm{WV}^{\prime}$ 's mental health community. W V Med J 1998;94:326-8.

6 Kellner R. Functional somatic symptoms and hypochondriasis: A survey of empirical studies. Arch Gen Psychiatry 1985;42:831-3.

7 Salkovkis PD, Clark DM. Panic disorder and hypochondriasis. Adv Behav Res Ther 1993; 15:23-48.

8 Kirmayer L, Taillefer FS. Somatoform disorders. In: Turner SM, Hersen M, eds. Adult psychopathlogy and diagnosis, 3rd ed. New York: John Wiley and Sons Inc, 1997;333-383.

9 Brown RJ. The cognitive psychology of dissociative states. Cognitive Neuropsychiatry 2002;7:221-35.

10 de Wet CJ, Mellers JDC, Garnder WN, et al. Pseudoseizures and asthma. J Neurol Neurosurg Psychiatry 2003;74:639-41.

11 Wolfe F, Hawley DJ. Evidence of disordered symptom appraisal in fibromyalgia: increased rates of reported comorbidity and comorbidity severity. Clin Exp Rheumatol 1999;17:297-303.

12 Cohen ME, Robins E, Purtell JJ, et al. Excessive surgery in hysteria. JAMA 1953;151:977-86.

13 Day Baird D, Dunson DB, Hill MC, et al. High cumulative incidence of uterine leiomyoma in black and white women: ultrasound evidence. Am J Obstet Gynecol 2003;188:100-7.

14 Simon GE, Gureje O. Stability of somatization disorder and somatization symptoms among primary care patients. Arch Gen Psychiatry 1999;56:90-5.

15 Carson AJ, Ringbauer B, Stone J, et al. Do medically unexplained symptoms matter? A prospective cohort study of 300 new referrals to neurology outpatient clinics. J Neurol Neurosurg Psychiatry 2000;68:207-10.

16 Lempert T, Dieterich M, Huppert T, et al. Psychogenic disorders in neurology: frequency and clinical spectrum. Acta Neurol Scand 1990;82:335-40. 